\title{
A New Model for Quantifying Anisotropic Scale Invariance and for Decomposition of Mixing Patterns ${ }^{1}$ Qiuming Cheng ${ }^{2,3}$
}

\begin{abstract}
A new power-law function has been derived to represent the relationship between area of the set consisting of wave numbers with spectral energy density above $S(A(>S))$ on the two-dimensional frequency plane and $S$. The power-law relation holds if the field concerned possessing isotropic scale invariance or generalized scaling invariance involves rotational and ratio-scale changing transforms. The equation is valid for dealing with common exploration geophysical and geochemical fields encountered in mineral exploration and environmental assessment. This power-law function not only provides a new model for characterizing anisotropic scaling invariance for generalized scaling field, for example, estimating the power exponent of power spectrum of generalized scale invariance measure in frequency domain, but also forms a theoretical base for the S-A filtering technique developed for decomposing a mixing field into components on the basis of distinct scaling properties in the frequency domain. It is demonstrated that the method has potential to become a general technique for image processing and pattern recognition.
\end{abstract}

KEY WORDS: multifractal, self-similarity, anisotropic scaling, geophysical and geochemical data processing, Landsat TM image.

\section{INTRODUCTION}

A two-dimensional (2D) multifractal measure may represent a field or pattern with scaling properties observed on or near the surface of the Earth or remotely sensed from space. The patterns may reflect variabilities projected on the Earth surface by multi-scale overlapping geological objects and superimposed processes occurring at or near the Earth's surface. The same type of phenomena can be found in other fields such as medical imaging where images are sensed from the outside of the human body and used to identify patterns pertaining to internal causes. Some of the useful signals can be weak and hidden within irrelevant patterns caused by features acting as noise, concealing useful information. Whether or not a real

\footnotetext{
${ }^{1}$ Received 5 September 2003; accepted 25 November 2003.

${ }^{2}$ Department of Earth and Amtospheric Science, Department of Geography, York University, Toronto, Ontario, Canada M3J 1P3; e-mail: qiuming@yorku.ca

${ }^{3}$ Earth Systems and Mineral Resources Lab, China University of Geosciences.
} 
component can be extracted from the total pattern and associated with a unique process (cause) or describe the probability distribution for a single regionalized random variable is obviously essential for pattern identification, and this is often related to the accuracy of decision making. Complexity involved in geoscientific spatial patterns is due to the variability, diversity, randomness, and temporal/space multiple-scale properties. The variability observed at the Earth's surface usually reflects multiple (cascade type) processes with multiple controling factors.

Spectral analysis has been widely used in physics for pattern recognition, signal processing, and decomposition. One of the common ways to convert between spatial domain and frequency domain is Fourier/Inverse Fourier transformation. Spectral energy density functions characterize the power spectrum distribution in the frequency domain. The advantage of dealing with fields in the frequency domain is that complex convolution operations in the spatial domain for correlation analysis, filtering and transformation can be simplified significantly in the frequency domain. Moreover, anisotropic spatial distribution of a spectral energy density retains the spatial structure of a field. Therefore, spatial analysis can be applied to anisotropic spectral energy density in the frequency domain in order to construct filtering functions to process the field (Cheng, 2001a,b; Cheng, Xu, and Grunsky, 1999, 2001). In this regard, to quantify anisotropy is essential for developing new techniques of pattern decomposition.

The relationships between multifractal modeling and spectral energy density functions may be useful for investigation of new methods of spatial-spectral analysis. The current paper has two purposes. Firstly, a new function relating multifractal model and spectral energy density function will be derived to show the powerlaw relationship, $A(>S) \propto S^{-2 / s}$ or $A(>S) \propto S^{-2 d / s}$, between the spectral energy density value $\left(S=\left\|F\left(\omega_{x}, \omega_{y}\right)\right\|\right)$ and "area" of the set with spectral energy density values above $S,\left\{\left(\omega_{x}, \omega_{y}\right):>S\right\}$, where $F$ represents Fourier transformation; $\omega_{x}$ and $\omega_{y}$ are wave numbers in horizontal and vertical directions, respectively; and $s$ and $d$ parameters to be introduced in the text. Secondly, it will introduce a new method based on the above model for separating geochemical and geophysical anomalies from background. Landsat TM images were used to validate the methods for identifying gold and copper associated alteration zones in the northwestern British Columbia, Canada.

\section{QUANTIFICATION OF ANISOTROPIC SCALE INVARIANCE}

Anisotropy is not only a common characteristic of geochemical and geophysical fields but also carries valuable information for image processing and pattern recognition. How to quantify and extract anisotropic properties is crucial for making use of anisotropy in data processing and classification. A number of authors have attempted to develop techniques for quantifying anisotropic properties. Fox and Hayes (1985) introduced different scaling exponents for different directions; 
Agterberg, Cheng, and Wright (1993) applied anisotropic transformation to locations of gold deposits to convert anisotropic to isotropic distributions; Schertzer and Lovejoy (1991) introduced the concept of generalized scale invariance; Lewis and others (1999) proposed a scale invariant generator technique; Cheng, Agterberg, and Ballantyne (1994) developed the $C-A$ method for quantifying anisotropy of geochemical data. In the current paper, a new technique is proposed to quantify anisotropy in the frequency domain. In comparison with most of the existing methods, the new method is particularly useful not only for quantifying anisotropy but also for constructing filters for decomposition of mixing patterns. A method developed on the basis of the new model will be discussed.

\section{Spectral Analysis of Isotropic Scale Invariance}

Scale invariance refers to the property of a system that does not change by changing scales. Properties at large-scale and small-scale are related by a scale changing transformation involving only the scale ratio. It can be proved that only the power-law function has the property:

$$
M(\delta)=c \delta^{-\beta}
$$

where $M$ is measure and $\delta$ is the measuring unit; $c$ and $\beta$ are two constants. The power-law function (1) is a one-dimensional (1D) function involving only one variable $\delta$. If this function is used to characterize $2 \mathrm{D}$ or $3 \mathrm{D}$ quantities it is usually assumed that the scaling is isotropic meaning the scale changing at the same ratio in different directions. Most of the existing fractal and multifractal modelling techniques are designed for isotropic scaling. For example, the commonly used box-counting method implies $N(\delta)=c \delta^{-D}$, where $N(\delta)$ is the number of boxes containing a feature; the boxes are usually squares with linear size $\delta$ and $D$ is the box-counting dimension. In the formulism of multifractal moment model, there is a power-law relationship between measure $\mu$ and measuring unit $\varepsilon, \mu(\varepsilon)=c \varepsilon^{\alpha}$, where $\mu(\varepsilon)$ is a measure defined in a square box of linear size $\varepsilon$ and $\alpha$ is the so-called singularity index. The power-law spectral energy density function often has the form:

$$
S(\omega)=c \omega^{-s}
$$

where $\omega=\left\|\left(\omega_{x}, \omega_{y}\right)\right\|$ is the norm of the wave numbers $\omega_{x}$ and $\omega_{y}$ for horizontal and vertical directions of the $2 \mathrm{D}$ field. $S(\omega)$ obtained by integrating the angular energy density. If this function is used to characterize an anisotropic 2D spectral energy density field, the anisotropy of the field will be "washed out" by the smoothing effect of the integral (Lewis and others, 1999). In order to introduce 
the new function to quantify anisotropy in the next section, here we first take a simple case by assuming an isotropic field so that Equation (2) holds true. Then the relationship between "area", $A(>S)$, in the frequency domain where the spectral energy density values are above a threshold $(S)$ and $S$ can be derived as follows

$$
A(>S)=\iint_{S(\omega)>S} d \omega_{x} d \omega_{y} \propto 2 \pi \int_{0}^{\left(\frac{S}{c}\right)^{-\frac{1}{s}}} \omega d \omega=\pi\left(\frac{S}{c}\right)^{-\frac{2}{s}} \propto S^{-\frac{2}{s}}
$$

using $\omega_{x}=\omega \sin \theta, \omega_{y}=\omega \cos \theta$ (coordinate transformation), and replacing $S(\omega)>$ $S$ by $\omega<(S / c)^{-1 / s}$. It can be seen that the function $A(>S)$ is a decreasing powerlaw function of spectral energy density value $S$ with exponent $-2 / s<0$. Comparison of Equations (2) and (3) suggests that Equation (2) holds true either because of the isotropic scaling measure so that it can be represented as a 1D function $S(\omega)$ or, otherwise, it washes out the anisotropy of the field by smoothing $S\left(\omega_{x}, \omega_{y}\right)$. Equation (3) does not have this problem although it was derived from (2) under the assumption of field isotropy. In the next section, the power-law relation (3) will be derived for a field with anisotropic scale invariance.

\section{Anisotropic Scale Invariance}

As discussed previously, isotropic scaling usually involves only one scalechanging factor as illustrated in (1). Since 1983, Schertzer and Lovejoy (1991) have developed the concept of general scale invariance (GSI), which defines the concept of scale in anisotropic scaling systems. In the formulism of GSI, taking 2D problem as example, the scaling in $x$ and $y$ directions will not be at the same scale-changing rate. Similarly, following the linear GSI formulism we can define the relationship between the measure and the measuring scale as

$$
M\left(T_{\lambda}\right)=M\left(\lambda^{-G}\right)=\lambda^{D}
$$

where $T_{\lambda}=\lambda^{-G}$ represents scale transformation, $G$ is a $2 \times 2$ matrix (for $2 \mathrm{D}$ problem), and $\lambda$ is scale ratio (generally $\lambda=$ initial unit $/ \delta$ ). If $G=\mathrm{I}$ as unity matrix, then the model (14) becomes the isotropic model (1). Otherwise, if $G$ is not unity, then model (4) characterizes anisotropic scale invariance. A general form of $G$ for a linear GSI was proposed by Lovejoy and Schertzer (1985) as

$$
G=d \mathbf{1}+c \mathbf{K}+f \mathbf{J}+e \mathbf{I}
$$


where: $\mathbf{1}, \mathbf{K}, \mathbf{J}$, and $\mathbf{I}$ are the bases of the $2 \mathrm{D}$ matrices

$$
\mathbf{1}=\left(\begin{array}{ll}
1 & 0 \\
0 & 1
\end{array}\right), \quad \mathbf{K}=\left(\begin{array}{cc}
1 & 0 \\
0 & -1
\end{array}\right), \quad \mathbf{J}=\left(\begin{array}{ll}
0 & 1 \\
1 & 0
\end{array}\right), \quad \mathbf{I}=\left(\begin{array}{cc}
0 & -1 \\
1 & 0
\end{array}\right)
$$

thus

$$
T_{\lambda}=\lambda^{-G}=\lambda^{d}\left(I \cosh (a u)-\frac{(G-d I)}{a} \sinh (a u)\right)
$$

where $u=\log \lambda$, and $\alpha^{2}=c^{2}+f^{2}-e^{2}$. If $\alpha^{2}<0$, the above formula holds but with $|\alpha|$ replacing $\alpha$. In the scale-changing operator, $d$ is a measure of overall contraction, $c$ is a measure of the relative scaling of the two coordinate axes, $f$ represents reflection across a line diagonal to the axes, and $e$ is a measure of rotation.

Various techniques have been developed for quantifying the anisotropic scale invariance under the formalism of GSI. The scale invariance generator (SIG) is one of several recently published techniques for dealing with anisotropic scale invariance (Lewis and others, 1999). In the following discussion, the relationship (3) will be explored on the basis of the linear GSI formalism relations (4)-(7).

Lovejoy and Schertzer (1985) have demonstrated that spectral energy density of a field with the generalized scaling invariance property under the formalism of GSI can be expressed as

$$
\left\langle S\left(\tilde{T}_{\lambda} \omega\right)\right\rangle=\lambda^{-s}\langle S(\omega)\rangle
$$

where $S$ represents spectral energy density, $\omega$ is wave number vector, $\tilde{T}_{\lambda}=\lambda^{\tilde{G}}$ is the scale-changing operator in Fourier space, $\tilde{G}$ is the generator in Fourier space, $\lambda$ is scale ratio and $s>0$ is the exponent. In the case of linear GSI, $\tilde{G}=G^{T}$, the transpose of the real space generator (5)-(7). The scale transformation $\tilde{T}_{\lambda}$ in the Fourier domain becomes (Lovejoy and Schertzer, 1985)

$$
\tilde{T}_{\lambda}=\lambda^{d}\left(I \cosh (a u)+\frac{\left(G^{T}-d I\right)}{a} \sinh (a u)\right)
$$

In order to derive the relation between $A(>S)$ and $S$ from Equation (9), the following discussion starts from transformation with operators $\mathbf{1}, \mathbf{K}, \mathbf{J}$, and $\mathbf{I}$. The primitive operators $\mathbf{1}, \mathbf{K}, \mathbf{J}$, and $\mathbf{I}$ transform $\omega\left(\omega_{x}\right.$ and $\left.\omega_{y}\right)$ by changing the scales with rotation and their combinations. Let us take any given contour with constant $S(\omega)$ and denote the set enclosed by the contour as $\Omega_{0}$ with "area" $A_{0}$, where the area has the unit of square wave numbers. It is noted that in the following discussion this "area" will be called area for convenience only. 
Denote the set transformed from the $\Omega_{0}$ after applying the scale-changing operator as $\Omega_{\lambda}=\tilde{T}_{\lambda} \Omega_{0}$. In order to derive the relationship between the area of $\Omega_{\lambda}\left(A\left(\Omega_{\lambda}\right)\right)$ and the area of $\Omega_{0}\left(A\left(\Omega_{0}\right)\right)$, we only need to find the general relationship for any circular set $\Omega_{0}$. For any circular set $\Omega_{0}$, its boundary can be expressed as $\partial \Omega_{0}=\left\{\omega:\left\|\omega-\omega_{0}\right\|=r\right\}$, where $\omega_{0}$ is the center of the set $\Omega_{0}$ and $r$ its radius. Therefore, $A\left(\Omega_{0}\right)=\pi\left\|\omega-\omega_{0}\right\|^{2}=\pi r^{2}$. Write $\omega_{\lambda}-\omega_{\lambda 0}=\tilde{T}_{\lambda}\left(\omega-\omega_{0}\right)$, then $\left\|\omega_{\lambda}-\omega_{\lambda 0}\right\|^{2}=\left(\omega-\omega_{0}\right)^{T} \tilde{T}_{\lambda}^{T} \tilde{T}_{\lambda}\left(\omega-\omega_{0}\right)=\left(\omega-\omega_{0}\right)^{T} A_{\lambda}\left(\omega-\omega_{0}\right)$, where $A_{\lambda}$ is a symmetrical matrix and with eigenvalues that can be proved to be $\lambda^{2(d-a)}$ and $\lambda^{2(d+\alpha)}$. Therefore, the area of the set $\left\{\omega_{\lambda}-\omega_{\lambda 0}=\tilde{T}_{\lambda}\left(\omega-\omega_{0}\right):\left\|\omega-\omega_{0}\right\|=r\right\}$ is equal to $\lambda^{2 d} A\left(\Omega_{0}\right)=\pi r^{2} \lambda^{2 d}$. Because the difference between the set $\left\{\omega_{\lambda}-\omega_{\lambda 0}=\right.$ $\left.\tilde{T}_{\lambda}\left(\omega-\omega_{0}\right):\left\|\omega-\omega_{0}\right\|=\mathrm{r}\right\}$ and $\Omega_{\lambda}$ only involves a translation operation, their areas must be equal. This can be expressed as

$$
A\left(\Omega_{\lambda}\right)=\pi r^{2} \lambda^{2 d}
$$

Because of the arbitrary choice of circle $\Omega_{0}$, relation (10) must hold true for any set $\Omega_{0}$. This is because any set of this type can be approximated by means of circles. The relation (10) is independent of $f, c$, and $e$ implying that the transformation involving rotation and symmetrical relative scaling of the two coordinate axes does not change the area.

Considering that the spectral energy density $S\left(\tilde{T}_{\lambda} \omega\right)$ decreases for increasing scale ratio $\lambda$, we can combine relations (10) with (8) which gives:

$$
A\left(\Omega_{\lambda}\right)=A(>S)=\pi r^{2}\left(S / S_{0}\right)^{-2 d / s} \propto S^{-2 d / s}
$$

where $\propto$ stands for "proportional to." Thus we have derived power-law relation between area $A(>S)$ and the threshold spectral energy density $S$. We can conclude that for isotropic scale invariance or anisotropic generalized scale invariance involving rotation and differential change of scales of two axes, the power-law relation (11) is exact. $2 d=$ Trace $(G)$ is called elliptical dimension. If $2 d=2$ corresponds to isotropic scale and in this case the relation (11) becomes the same form as (3). Most of the geophysical and geochemical fields for mineral exploration reflecting the geological bodies formed through overlap of multiphase rotational and relative compression deformation, therefore the relation (11) holds true for these data. This relation will be used as the foundation for the $S-A$ method for decomposing mixing patterns as discussed in the next section.

It can be seen that the result (11) is independent of $\alpha^{2}=f^{2}+c^{2}-e^{2}$. In addition, the area $(A)$ and power spectrum density $S$ in the Equation (11) are real values even if $\alpha^{2}<0$. This property makes the Equation (11) useful in dealing with complex power spectrum where $\alpha^{2}<0$ and matrix $G$ has complex eigenvalues and the measure gives log-log oscillations in power spectrum. 
It can be seen that although the derivation of Equation (11) was based on the linear GSI transformation matrix $G$, the result holds true for nonlinear GSI as well. As suggested by Lovejoy in his critical reviewer's comments the Equation (3) can be abstracted as follows:

We can use the scale function $\omega_{\lambda}$ and the generalized angle $\theta$, then the following area element can be used in integral to express $A\left(\Omega_{\lambda}\right)$

$$
\mathrm{d} \omega_{\lambda}=d\left\|\omega_{\lambda}\right\|^{D} d \theta
$$

where $D$ is the elliptical dimension. Equation (12) could be regarded as the definition of $\theta$; it will depend on the exact scale function used. If a scale function exists, then we can obtain the area less than scale $\left\|\omega_{\lambda}\right\|$ :

$$
A\left(\Omega_{\lambda}\right)=\iint_{\left\|\omega_{\lambda}^{\prime}\right\|<\left\|\omega_{\lambda}\right\|} d\left\|\omega_{\lambda}^{\prime}\right\|^{D} d \theta=\frac{\Omega}{D}\left\|\omega_{\lambda}\right\|^{D}
$$

where $\Omega$ is the "total angle" ( $=2 \pi$ in $2 \mathrm{D}$ isotropic system). The corresponding generalized scaling for the power spectral density

$$
\left\langle S\left(\omega_{\lambda}\right)\right\rangle=\left\|\omega_{\lambda}\right\|^{-s}
$$

Combining Eqns. (13) and (14) we obtain

$$
A\left(\Omega_{\lambda}\right)=\frac{\Omega}{D} S^{-\frac{D}{s}}
$$

\section{DECOMPOSITION OF MULTIFRACTAL MEASURES}

Since power-law relation (11) holds true for anisotropic fields, it is useful in practice to characterize the spatial distribution of a power-spectrum. For example, it can be used to test whether a field possesses one single power-law relation for all ranges of spectral energy density or if several power-law relations may be needed to fit the relation (11). In the latter case one can determine a threshold in the frequency domain to construct different filters to decompose the field in the space domain.

\section{Decomposition of Patterns}

Assume the observed field $T(x, y)$ represents bulk value of element concentration in surface media such as rock samples created by various large-scale 
geological processes and small-scale mineralization. These two different processes may cause distinct distributions of the same element concentration in the same area to be denoted as $B(x, y)$ and $A(x, y)$, which are unknown components of $T(x, y)$. The mixing could be of two different forms: product or sum. By log-transformation the former becomes the latter. Therefore, only the sum mixing effect (summation form) will be discussed. It can be expressed as

$$
T(x, y)=B(x, y)+A(x, y)
$$

where $T(x, y)$ represents bulk value observed at location $(x, y)$ in an area, $B(x, y)$ the component reflecting regional large-scale processes, and $A(x, y)$ the component reflecting local small-scale processes. There are a number of techniques available for decomposing $T$ into $A$ and $B$. For example,

(1) Threshold-based method that separates $T$ into $B$ and $A$ on the basis of value $T$ in comparison with the threshold $T_{0}: B=T$ if $T<T_{0}$ and otherwise $A=T$ if $T \geq T_{0}$. There have been a number of ways to set thresholds such as $T_{0}=E(T)+\alpha S(T)$, where $E$ and $S$ stand for mean and standard deviation of the $T$ and $\alpha$ is the factor. This method assumes that $B$ and $A$ have separate value populations.

(2) Methods such as trend analysis set $B$ as constant or as a polynomial surface and the residuals as $A$. These types of methods use predetermined polynomial surfaces to fit $B$, which may be applicable in special situations where $\mathrm{B}$ shows regional variability that can be approximated by predetermined polynomial surfaces.

(3) Methods such as kriging take $B$ as the mean surface for normally and log-normally distributed regionalized random variables and the residuals as $A$. This treatment depends on choice of spatial association model.

(4) Frequency-based filter techniques separate $T$ into $B$ and $A$ based on frequency periodic properties, such as low- and high-pass filtering and band-pass filtering. These types of methods separate $T$ into $B$ and $A$ both with distinct frequency or wavelength properties.

(5) The method to be introduced in detail below separates $T$ into $B$ and $A$ based on distinct anisotropic scaling properties in the frequency domain.

\section{Fractal Filtering Technique $(S-A)$}

The $S-A$ method developed by Cheng, Xu, and Grunsky (1999) constructs fractal filters on the basis of distinct power-laws determined by fitting different relations (11). Usually, several straight-line segments can be fitted to the relation (11) on log-log plot. Each range of spectral energy density within which relation 
(11) holds true can be used to define a filter. For example, if two straight-line segments are fitted to the data, and these straight lines yield the threshold $S_{0}$, then two filters can be defined as: $G_{B}(\omega)=1$ if $S(\omega)>S_{0}$ and otherwise $G_{B}(\omega)=0$; and the second one with $G_{A}(\omega)=1$ if $S(\omega) \leq S_{0}$ and otherwise $G_{A}(\omega)=0$. From the definition of $G_{A}(\omega)$ and $G_{B}(\omega)$, we can see that the shapes of the filters could be irregular depending on the complexity of the spectral energy density distribution. However, in general, the wave numbers $\omega$ in filter $G_{A}(\omega)$ are relatively larger than those in $G_{B}(\omega)$ implying that the frequency in $G_{A}(\omega)$ is relatively higher than those in $G_{B}(\omega)$. In this sense, $G_{A}(\omega)$ corresponds to a relatively high frequency component and $G_{B}(\omega)$ to a relatively low frequency component. However, one must keep in mind that the two filters are not sharply bounded either by frequency or by wave numbers. They are defined in such a way that the spectral energy density distributions on the two filters satisfy distinct power-laws or have different anisotropic scaling properties, that in a simple situation, may correspond to distinct (isotropic) self-similarities or self-affinities (stratification). Applying the Inverse Fourier Transformation with these two filters applied to the Fourier transformed functions we can get decomposed components in the space domain:

$$
\left.\left.B=F^{-1}\left[F(T) G_{B}\right)\right], A=F^{-1}\left[F(T) G_{A}\right)\right]
$$

where $F$ and $F^{-1}$ represent the Fourier and Inverse Fourier transformations of $T$, respectively. Therefore, the two components $B$ and $A$ have different properties. The frequencies on $B$ and $A$ may not be totally different but they must show distinct scaling properties in their spatial distribution as quantified by two distinct power-laws in the frequency domain.

\section{A CASE STUDY}

The data to be used to validate the relation (11) and to demonstrate the application of the $S-A$ method are Landsat TM images, received on 9 September 1985 , covering the Mitchell-Sulphurets mineral district, northwestern British Columbia. These images have been studied for alteration identification (Cheng, 1999; Rencz, Harris, and Ballantyne, 1994) and for nonlinear modeling (Cheng 1999). The dataset consists of seven TM images (bands 1-7) with 30-m resolution for bands $1-5$ and 7 (120 $\mathrm{m}$ for band 6$)$ each of which contains $496 c \times 777 r$ pixels covering an area of about $350 \mathrm{~km}^{2}$.

The relations (11) and (13) will be applied to TM bands 1 to 5 and 7 only for maintaining a uniform resolution. Figure 1 shows the TM bands 1 and 7 (other images not shown here). The spectral energy density values $(S)$ obtained by means of the Fast Fourier transform in GeoDAS GIS (Cheng, 2000) are shown in Fig. 2. The centers of the maps in Fig. 2 correspond to $\omega=0$. The values of $S$ towards 


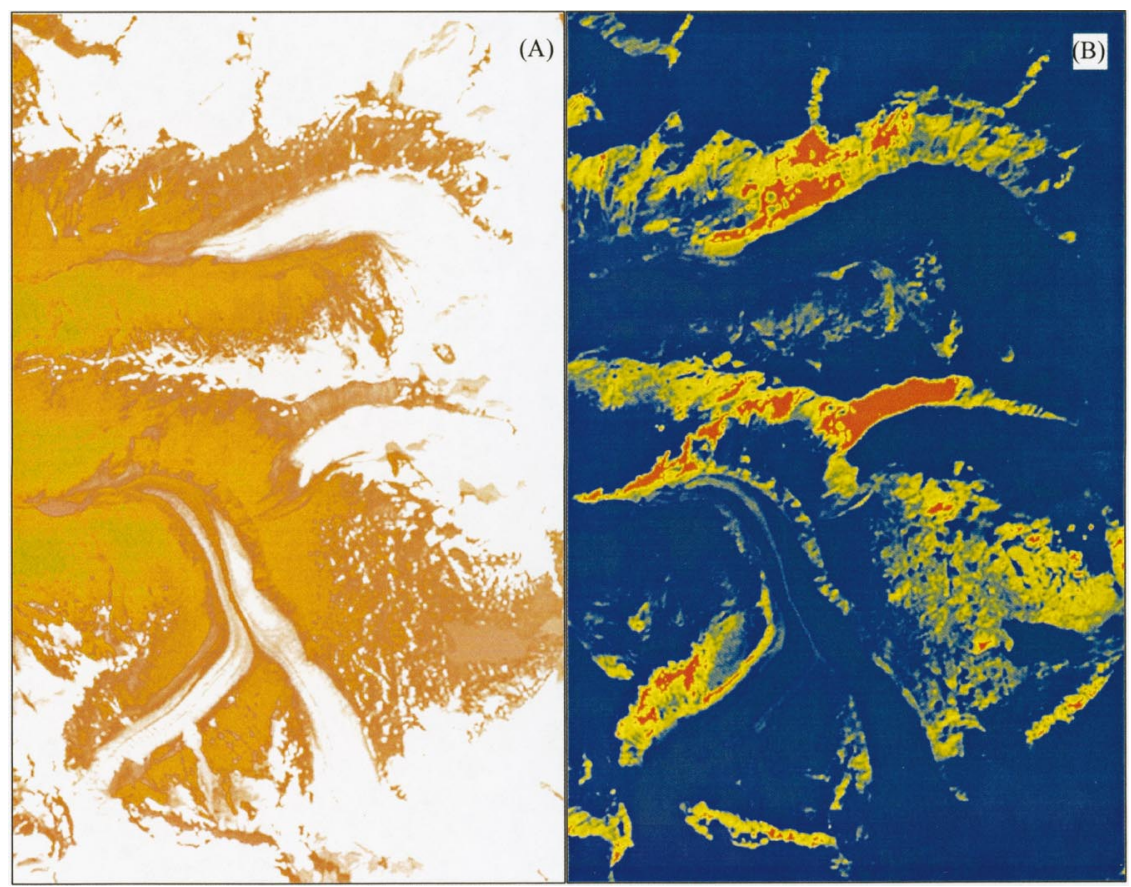

Figure 1. Landsat TM image bands $1(A)$ and $7(B)$, containing $496 c \times 777 r$ pixels at 30 -m resolution covering an area of about $350 \mathrm{~km}^{2}$, received on 9 September 1985, from Mitchell-Sulphurets area, northwestern British Columbia (Rencz, Harris, and Ballantyne, 1994).

the center are generally higher than the values away from the center. The image is diagonally symmetrical. From all the spectral energy density images calculated for the six TM bands (results for bands 2-5 not shown), we can see that the spectral energy density images for bands $1-4$ are $N-S$ elongated and are symmetrical both vertically and horizontally. Whereas the spectral energy density images for bands 5 and 7 are elongated in the north-north-western (NNW) direction with a few degree departure from north-south orientation. The two vertical and horizontal strips on Fig. 2 are due to edge effects. Because the constant areas occupied by the two strips are relatively small, it does not seriously affect the power-law plot between $A(>S)$ and $S$. The effect can be corrected by applying a smoothing transformation or through other edge effect treatment (more discussion about edge effect correction can be found in $\mathrm{Ge}$, Cheng, and Zhang (in press). The results of $A(>S)$ versus $S$ obtained for the six images are shown in Figs. 3(A)-(F), respectively. When the data are plotted at log-log scale, towards the right the value $S$ increases and accordingly the frequency decreases. On the farmost right the plot becomes flat because of the area increment becoming zero. To the left the plot tends towards 


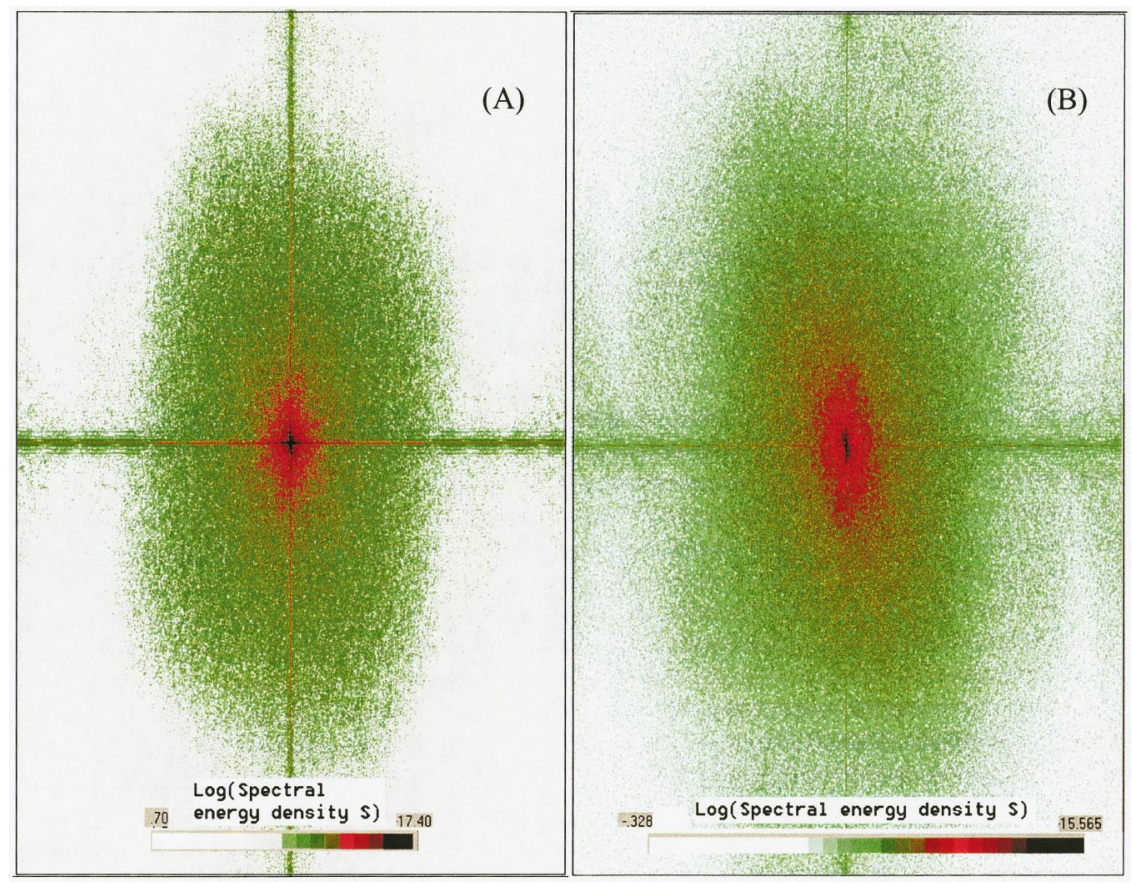

Figure 2. Images showing distribution of spectral energy density values as two-dimensional function of wave numbers in vertical and horizontal directions. The center of the image is the origin where wave number equals zero. The spectral energy density function was obtained by Fast Fourier transform. (A) The result obtained for image band 1 shown in Fig. 1(A) and (B) result obtained for image band 7 shown in Fig. 1(B).

relatively low value $S$ and high frequency. A few points on the plot from the farmost left are of large uncertainty because of the high frequency energy density function. Therefore, the points on both the farmost right and left ends should not be included in the straight-line fitting. The data of $A(>S)$ and $S$ obtained for the six images on Fig. 3 can be generally fitted by two straight-line segments using the least-square method. The bands 1-4 images show relatively short segments on the right and most values of $S$ can be fitted as one straight line. On the other hand, the bands 5 and 7 images show two different straight-line fittings.

Cheng (1999) has found that the patterns defined on the basis of bands 1-4 are close to single fractals whereas the patterns on bands 5 and 7 are multifractals. The current study has demonstrated bands 5 and 7 show two significantly different power-law relations between $A(>S)$ and $S$ whereas bands $1-4$ show only one significant relation. For this reason, the $S-A$ method is used only to decompose the images of bands 5 and 7 into two components on the basis of these two distinct power-law models. For example, in Fig. 3(F) the two straight lines separate the 
spectral energy density values into two ranges with cutoff value $\log \left(S_{0}\right)=12.47$ or $S_{0}=261904$, where Log is natural $\log$. The first filter can be defined as $G_{B}(\omega)=1$ if $S(\omega)>S_{0}$ and, otherwise, $G_{B}(\omega)=0$. Similarly, the other filter can be defined as $G_{A}(\omega)=1$ if $S(\omega)<S_{0}$ and, otherwise, $G_{A}(\omega)=0$. The shapes of these two filters are irregular. The norm of wave number vector $\|\omega\|$ in $G_{B}$ is generally small in comparison with that in $G_{A}$; however, because of their irregular shapes, these two filters are not sharply bounded by wave number. Therefore, although the frequency in $\mathrm{G}_{B}$ is generally lower than that in $G_{A}$, these filters are not sharply
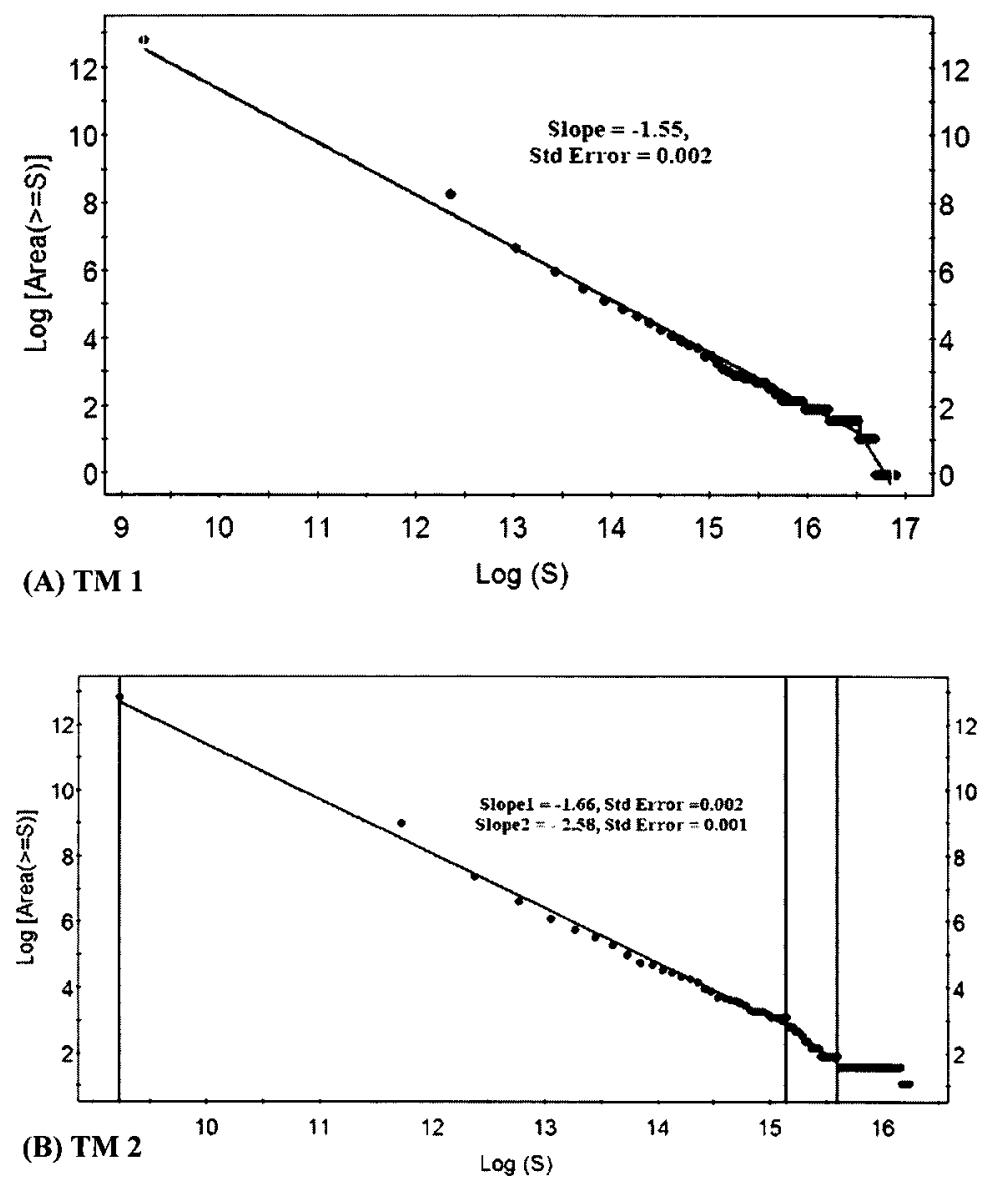

Figure 3. Plots showing the relationship between $A(>S)$ and $S$ obtained for images bands $1-5$ and 7 , respectively. The log-transformation is natural $\log$. The dots represent spectral energy density values at equal interval with 100 classes. The low and high values were set at about low and up 3\% pixels as bounds for display purposes. Two straight-line segments were fitted by Least-Squares. Vertical lines represent the dividers. 

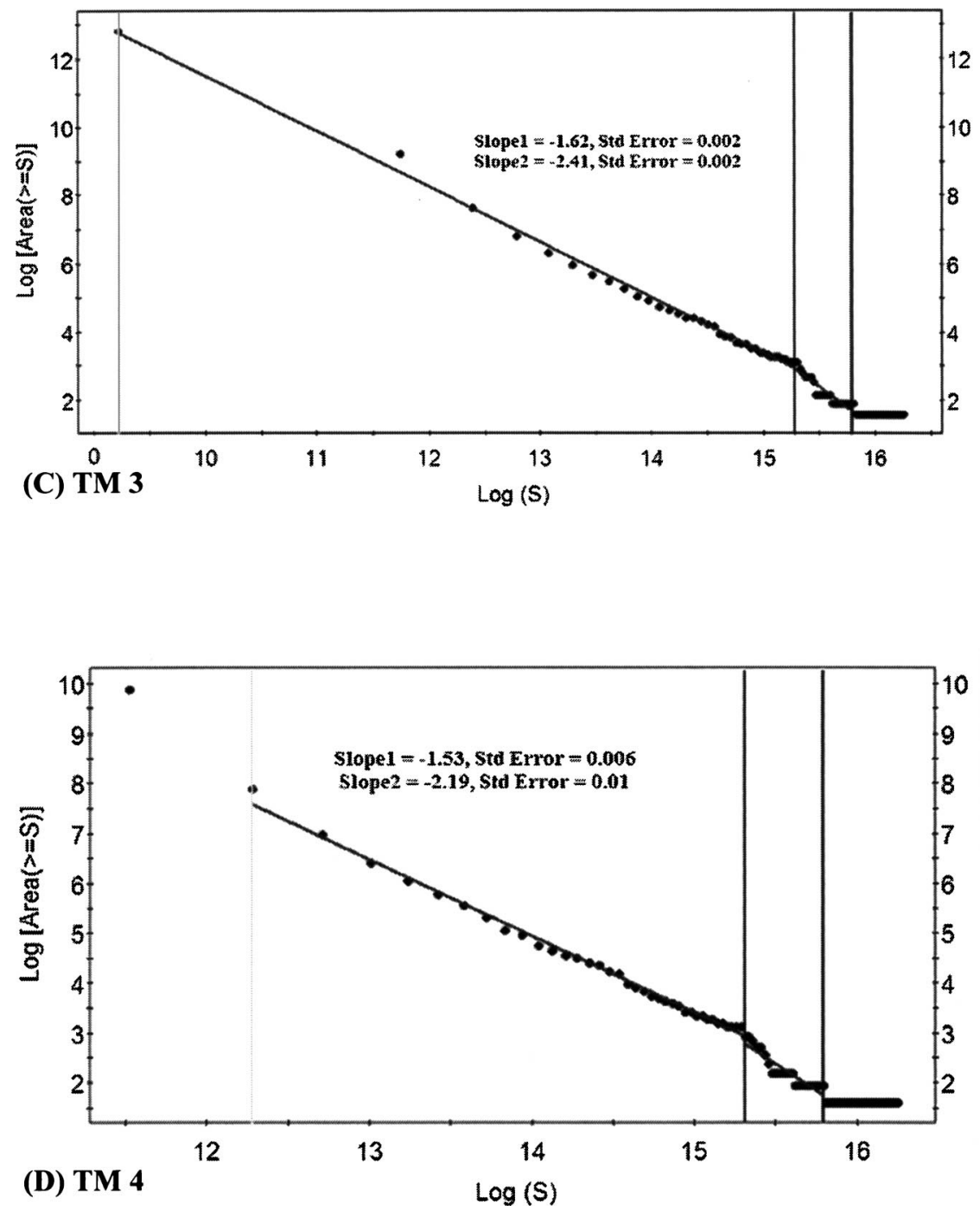

Figure 3. (Continued)

bounded by frequency. This is a fundamental distinction between the $S-A$ method and other frequency-based regular filtering techniques including high-, low-, and band-pass filters.

The signals in the filters constructed on the $S-A$ plot are not only based on magnitude of frequency but also on the power-law property of the spectral energy density distribution. In this regard, $S-A$ can be considered as a spectral-spatial 

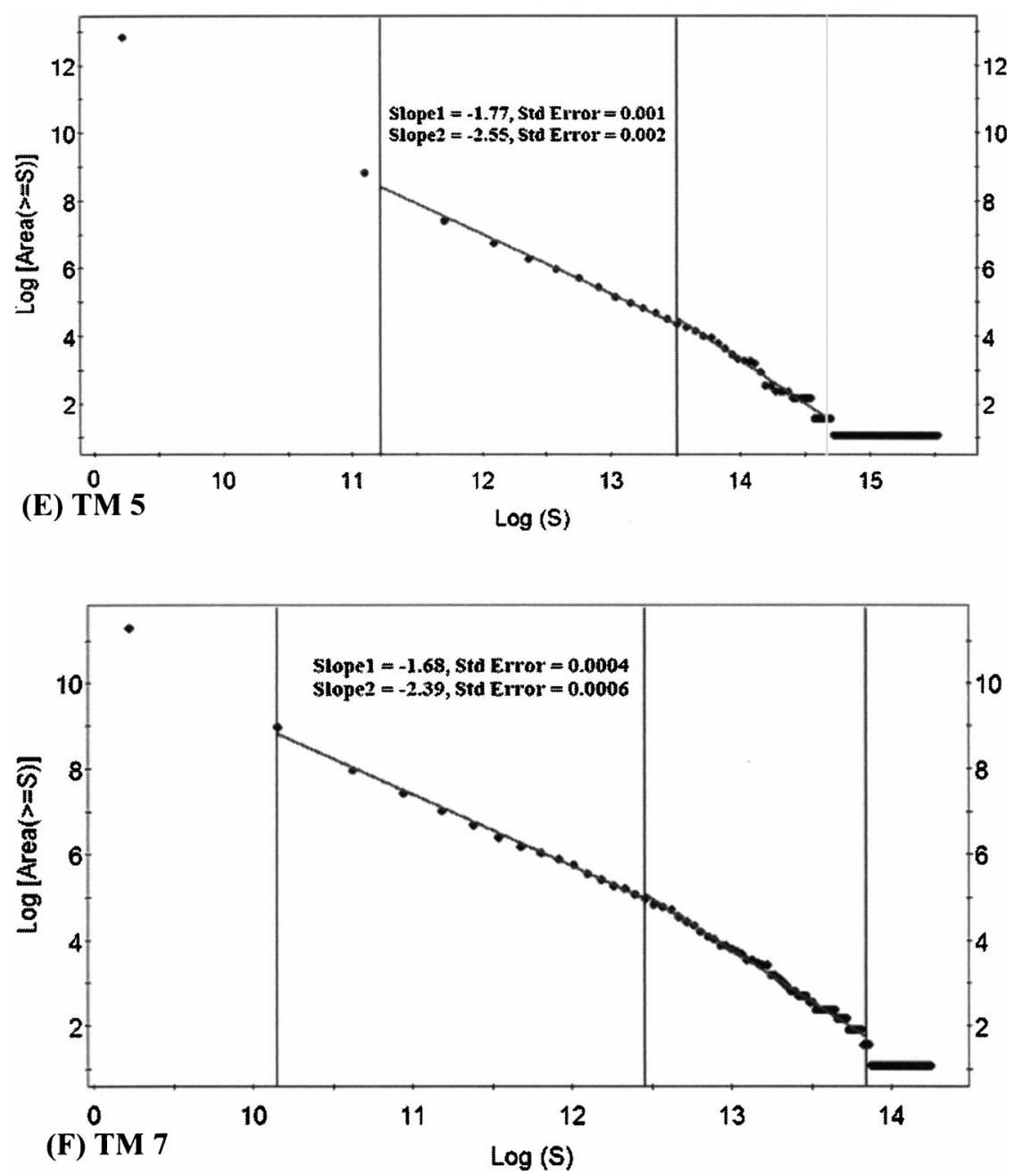

Figure 3. (Continued)

analysis method rather than a frequency-based filter technique. The two decomposed components of band 7 with $G_{B}$ and $G_{A}$ are shown in Figs. 4(A) and (B), respectively. The component obtained from applying $G_{B}$ in Fig. 4(A) shows general background variability of the image with high values reflecting outcropping rocks and alteration zones as well as relatively high topographical areas. The component obtained from applying $G_{A}$ in Fig. 4(B) shows general residuals of the image with high values reflecting detail patterns of both alteration zones and altered rocks between glacial deposits. Comparing the component in Fig. 4(B) with 


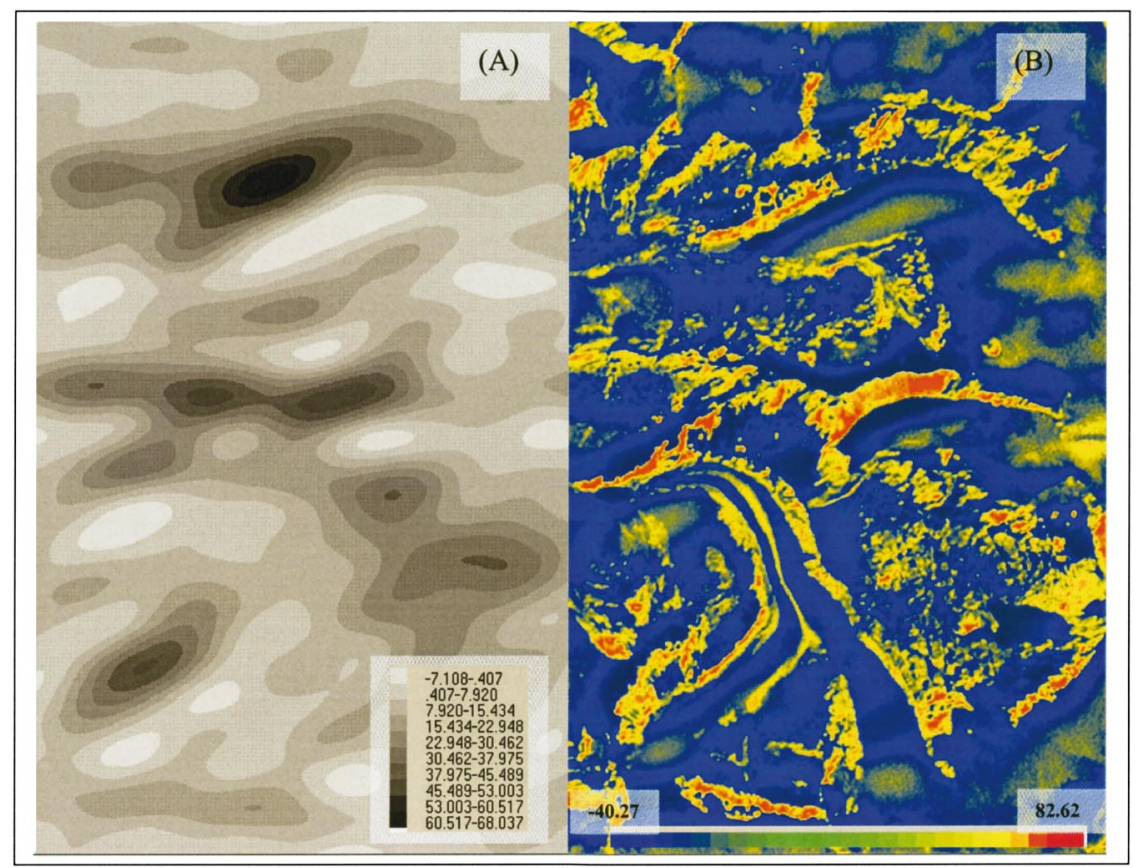

Figure 4. Plots showing the decomposed image from image band 7 (Fig. 1(B)) by Inverse Fourier transformation with the filters defined on the basis of the straight-line fitting on Fig. 3(F) (see text for details).

the original image in Fig. 1(B) we can see that the decomposed image shows the patterns (alteration zones and altered rocks between glacial deposits) in great detail. More geological interpretation will be discussed in a separate paper.

\section{CONCLUSIONS}

From a multifractal point of view, the spatial autocorrelation, and spectral energy density are related to the low-order moment statistics of multifractal model. The spectral energy density function and spatial autocorrelation functions can be used to quantify anisotropic property of field from the frequency and space domains, respectively. The new power-law relationship $A(>S)$ versus $S$ derived in the current paper can be used as a general model to quantify anisotropic scale invariance of a field because of differential contraction and rotational operation. The new technique ( $S-A$ method) has been demonstrated to be useful for decomposing mixing patterns on the basis of distinct scaling properties in the frequency domain. It has potential to become a general technique for image processing and pattern recognition. 


\section{ACKNOWLEDGMENTS}

Thanks are due to Dr. S. Lovejoy and Dr. F. P. Agterberg for critical reading of the paper and constructive comments. Dr. S. Lovejoy suggested an improved derivation of Eq. (15). Part of this paper was presented at the international workshop on "Non-traditional Mineral Resources" held in China University of Geosciences, Beijing, August 15-16, 1999. The research was jointly supported by "nonlinear information extraction and integration" a Chinese 863 Program; NSERC Discovery Research "development of GIS technology for mineral exploration and environmental assessments" (ERC-OGP0183993) and Ontario Mineral Exploration Technology (OMET) grant.

\section{REFERENCES}

Agterberg, F. P., Cheng, Q., and Wright, D., 1993, Fractal modeling of mineral deposits, in Proceedings XXIV APCOM, October 31-November 3, 1993, Montreal, Quebec, v. 1 p. 43-53.

Cheng, Q., 1999, Multifractality and spatial statistics: Comp. Geosci., v. 25, no. 10, p. 8-25.

Cheng, Q., 2000, GeoData Analysis System (GeoDAS) for Mineral Exploration: User's Guide and Exercise Manual, in Unpublished notes for the training workshop on GeoDAS held at York University, November 1-3, 2000, 204 p. (www.gisworld.org/geodas).

Cheng, Q., 2001a, Spatial self-similarity and geophysical and geochemical anomaly decomposition: J. Geophys. Prog. J. Chin. Acad. Sci., v. 16, no. 2, p. 8-17. (in Chinese with English Abstract).

Cheng, Q., 2001b, Self-similarity/self-affinity and pattern recognition techniques for GIS analysis and image processing, in IAMG2001 Meeting, Cancún, Mexico, September 6-12, 2001 (6 pages on CD).

Cheng, Q., Agterberg, F. P., and Ballantyne, S. B., 1994, The separation of geochemical anomalies from background by fractal methods: J. Geochem. Explor. v. 51, no. 2, p. 109-130.

Cheng, Q., Xu, Y., and Grunsky, E., 1999, Integrated spatial and spectrum analysis for geochemical anomaly separation, in Lippard, J. L., Naess, A., and Sinding-Larsen, R., eds., Proceedings of the International Association of Mathematical Geology Meeting, Trondheim, Norway I, p. 87-92.

Cheng, Q., Xu, Y., and Grunsky, E., 2001, Multifractal power spectrum-area method for geochemical anomaly separation: Nat. Resour. Res., v. 9, no. 1, p. 43-51.

Fox, C. G., and Hayes, D., 1985, Quantitative methods for analyzing the roughness of the sea floor: Rev. Geophys., v. 23, p. 1-48.

Ge, Y., Cheng, Q., and Zhang, S., in press, Edge effect correction in S-A method for geochemical anomaly separation: J. Earth Sci.

Lewis, G. M., Lovejoy, S., Schertzer, D., and Pecknold, S., 1999, The scale invariant generator technique for quantifying anisotropic scale invariance: Comp. Geosci., v. 25, no. 9, p. 963-978.

Lovejoy, S., and Schertzer, D., 1985, Generalized scale invariance and fractal models of rain: Water Resour. Res., v. 21, no. 8, p. 1233-1250.

Rencz, A., Harris, J., and Ballantyne, B. B., 1994, Landsat TM imagery for alteration identification, in Current research 1994E, Geological Survey of Canada, Ottawa, p. 277-282.

Schertzer, D., and Lovejoy, S. (Eds.), 1991, Nonlinear Variability in Geophysics: Kluwer Academic, Dordrecht, The Netherlands, 318 pp. 